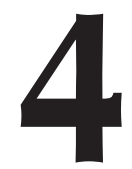

\title{
LAS CONCEPCIONES PEDAGÓGICAS DEL PROFESORADO UNIVERSITARIO: UN PUNTO DE PARTIDA PARA EL CAMBIO DOCENTE
}

\author{
(PEDAGOGICAL CONCEPTIONS OF UNIVERSITY TEACHING: A STARTING \\ POINT FOR TEACHER CHANGE)
}

José Antonio Pineda-Alfonso

Olga María Duarte Piña

Universidad de Sevilla

DOI: $10.5944 / e d u c X X 1.25409$

\section{Cómo referenciar este artículo/How to reference this article:}

Pineda-Alfonso, J. A. y Duarte Piña, O. M. (2020). Las concepciones pedagógicas del profesorado universitario: un punto de partida para el cambio docente. Educación XX1, 23(2), 95-118, doi: 10.5944/educXX1.25409

Pineda-Alfonso, J. A. \& Duarte Piña, O. M. (2020). Pedagogical conceptions of university teaching: a starting point for teacher change. Educación XX1, 23(2), 95-118, doi: 10.5944/ educXX1.25409

\section{RESUMEN}

Presentamos una investigación realizada en el marco de un programa pionero que viene desarrollándose en los últimos años en la Universidad de Sevilla cuya finalidad es conocer las concepciones que los participantes tienen de su docencia para hacerlas evolucionar hacia un modelo centrado en el aprendizaje. En este artículo abordamos un estudio sobre las concepciones que tienen los docentes sobre su propia disciplina y sobre el aprendizaje de los estudiantes, así como sobre las creencias ligadas a la construcción de su identidad profesional docente antes de iniciar el programa formativo. Hemos utilizado un cuestionario de respuestas abiertas y se han codificado éstas utilizando un sistema de categorías con una hipótesis de progresión de tres valores. Los resultados muestran una clara predominancia de las unidades de información de menor nivel de complejidad, en las que los 
docentes se identifican preferentemente con su labor como investigadores y consideran la docencia una obligación académica. Paralelamente, su concepción de la docencia se centra en la enseñanza y atribuyen escaso valor a la participación de los alumnos en el proceso de aprendizaje. En cuanto a la concepción de la propia disciplina, encontramos evidencias de una concepción absolutista y estática del conocimiento científico sin implicaciones sociales. Junto a esto aparecen unidades de información más evolucionadas, que ponen de manifiesto la posibilidad de transiciones a concepciones más complejas. Las conclusiones del estudio nos muestran vías posibles y útiles para orientar itinerarios formativos del profesorado universitario.

\section{PALABRAS CLAVE}

Enseñanza superior; formación de profesores; concepciones epistemológicas; proceso de aprendizaje; identidad docente.

\section{ABSTRACT}

We present a research project which has been carried out within the framework of a pioneering program that has been developed in recent years at the University of Seville, the aim of which is to understand the participants' conceptions of their teaching in order to make them evolve towards a model centred on learning. In this article we approach a study of the conceptions of the discipline itself and of the learning that teachers have, as well as on their beliefs linked to the construction of their professional teaching identity before starting the training program. We have used an open-ended questionnaire and these have been coded using a system of categories with a progression hypothesis of 3 values. The results show a clear predominance of information units with a lower level of complexity, where teachers preferably identify with their work as researchers and consider teaching to be an academic obligation. At the same time, their conception of teaching focuses on teaching and places little value on the participation of students in the learning process. As for the conception of the discipline itself, we find evidence of an absolutist and static conception of scientific knowledge without social implications. Along with this, more evolved units of information appear, which reveal the possibility of transitions to more complex conceptions. The conclusions of the study show us possible and useful ways to guide training itineraries for university teachers. 


\section{KEY WORDS}

Higher education; teacher training; epistemological conceptions; learning process; teaching professional identity.

\section{INTRODUCCIÓN}

Desde el curso 2013/2014 se viene desarrollando en la Universidad de Sevilla un programa de formación del profesorado orientado a la mejora de su propia práctica. Este programa se conoce como Programa de Formación e Innovación Docente del Profesorado Universitario (FIDOP) y consta de varias etapas en las que los formadores conducen y acompañan a los participantes en un trabajo basado en la descripción de sus clases, el análisis de las actividades que realizan y la reflexión sobre su modelo metodológico habitual, planteando un modelo metodológico que posibilite las mejoras de su docencia mediante Ciclos de Mejora Docente (CMD). La orientación del formador es fundamental tanto en los procedimientos de trabajo como en la aportación de nuevas perspectivas y enfoques para abordar los problemas y obstáculos, respetando que las decisiones finales, sobre la dirección y la intensidad de las innovaciones, son responsabilidad exclusiva de cada participante.

Los CMD obedecen a la lógica de la investigación-acción (Stenhouse, 1985) y suponen la reflexión sobre y en la propia práctica (Schön, 1992), representando uno de los ejes de acción fundamentales del programa FIDOP en los que se establece la conexión de la formación docente con la práctica diaria en el aula, que además se ha revelado como una fórmula eficaz para el cambio metodológico y para el fomento de la construcción de una nueva identidad profesional docente (Conde-Jiménez y Martín-Gutiérrez, 2016; Vázquez, Solís y Porlán, 2017).

En este sentido, los principios generales del programa son:

- El aula es el centro de la mejora docente.

- Incrementar la calidad de la docencia es un proceso gradual que requiere tiempo, constancia, acompañamiento experto y apoyo entre iguales.

- El cambio requiere de una estrategia que vincule formación e innovación docente.

- La mejora docente de calidad ha de basarse al mismo tiempo en la implicación activa del profesorado y en el conocimiento experto. 
- La estrategia de cambio busca un efecto progresivo que va concretándose en la aplicación de cada Ciclo de Mejora y la posterior reflexión, comunicación y publicación del mismo en las Jornadas de Formación e Innovación Docente del Profesorado Universitario de la Universidad de Sevilla (http://dx.doi.org/10.12795/JDU).

En coherencia con un conjunto de investigaciones internacionales que vienen indagando sobre los obstáculos a los que se enfrentan los participantes en distintos programas de formación del profesorado universitario (Gibbs \& Coffey, 2004), y las estrategias más efectivas para su mejora (Bain, 2004), se están analizando los resultados del programa FIDOP a través del Proyecto I+D+i La Formación Docente del Profesorado Universitario. Progresos y Obstáculos de los Participantes en un Programa Basado en Ciclos de Mejora de su Práctica (EDU2016-75604-P). En este marco, nuestro estudio abordará las concepciones iniciales de los profesores universitarios participantes sobre su identidad profesional, su propia disciplina de referencia y el valor social de la misma, y sobre cómo aprende su alumnado, pues estudios recientes han puesto de manifiesto que estas tres dimensiones son muy relevantes para el cambio profesional del docente universitario y para el diseño de propuestas formativas orientadas a este fin (Monereo, 2014; Paricio Royo, Fernández March y Fernández Fernández, 2019).

\section{FUNDAMENTACIÓN TEÓRICA}

Diversas investigaciones realizadas en los últimos 30 años señalan que la mayoría de los profesores universitarios no tienen las habilidades necesarias para realizar adecuadamente sus tareas de enseñanza y muy poco conocimiento sobre prácticas de enseñanza efectivas (Amundsen \& Wilson, 2012). Además, en España, distintos trabajos muestran que el porcentaje de profesores que utiliza el método tradicional sigue siendo mayoritario, a pesar de que la enseñanza centrada en el estudiante parece más eficaz (Zabalza, Cid y Trillo, 2014).

En el ámbito universitario, el número de investigaciones que tratan sobre la mejora de la docencia se ha incrementado, y a ello se han sumado las exigencias del Espacio Europeo de Educación Superior, volcadas hacia la conveniencia de que el profesorado universitario tenga una formación pedagógica, desarrolle un modelo de docencia centrado en el estudiante y establezca una interrelación entre la investigación y la docencia (Medina Moya y Jarauta Borrasca, 2013). En esta línea se inscriben las recientes recomendaciones para la universidad del siglo XXI, donde se prioriza el aprendizaje "en menoscabo de la enseñanza como elemento definidor de la misión institucional” (Zabalza et al., 2014, p. 42) y la necesidad de mejorar 
el pensamiento y las habilidades pedagógicas del profesorado universitario, reconociendo que en este campo las investigaciones son insuficientes (Martín del Pozo, Pineda-Alfonso y Duarte, 2017).

Centrándonos en las dimensiones de análisis que son objeto de la presente investigación, concepción de la propia disciplina, concepción del aprendizaje e identidad profesional, han de tenerse en cuenta las particularidades del profesorado universitario, que no encontramos en otros niveles educativos, pues "se trata de un profesorado en cuya formación se ha priorizado la especialización en un ámbito disciplinar, lo que hace que esté más orientado a la disciplina que a la propia profesión docente" (Zabalza et al., 2014, p. 41). Además, afirma Postareff (2007) que lo que caracteriza al profesorado universitario es el dominio de su propia disciplina, incluida su experiencia en torno a este dominio en relación con las concepciones que los docentes tienen sobre su modelo didáctico. Este modelo didáctico estaría formado por "pautas, rutinas y esquemas de acción que no responden a decisiones fundamentadas sino a hábitos asumidos de manera poco consciente" (De Alba y Porlán, 2017, p. 37) y que lleva a la repetición de comportamientos basados en la imitación de modelos o referentes sin la debida reflexión sobre sus fundamentos y sus implicaciones para una mejora de la enseñanza y el aprendizaje de los estudiantes.

Por ello, una variable fundamental en la definición de la concepción del aprendizaje es el conocimiento de la propia disciplina académica de referencia. En este sentido, una concepción crítica y profunda de la propia disciplina por parte del docente, incluyendo su evolución histórica y sus implicaciones sociales, favorecería procesos de aprendizaje en los que el estudiante adquiera una comprensión compleja del conocimiento que le permita el desarrollo de competencias profesionales de alto nivel (Paricio Royo et al., 2019).

Con respecto a la concepción de la identidad profesional docente, hay tres dimensiones que la determinan: la primera de ellas se refiere al rol docente y a sus concepciones sobre cómo enseñar, aprender y evaluar; la segunda alude a los cambios en las estrategias de enseñanza, aprendizaje y evaluación, y la tercera tiene en cuenta el cambio que se produce en las emociones de los docentes asociadas a su labor y la interpretación que hace de la misma (Cfr. Monereo, 2014, pp. 55-58). Pero no siempre el modelo didáctico personal implica una identidad, ni esta se conforma en un proceso espontáneo, sino más bien es una construcción que resulta de la incorporación desde las experiencias previas a ser docente. Por tanto, "la mayoría de los docentes declara inspirarse en su propia historia como estudiantes (o de sus pares), sin profundizar, en general, en elementos teóricos que puedan dar sentido a esta racionalidad pedagógica" (Drago, Espejo y González, 
2015, p. 100); más bien parece ser que es en la cotidianeidad del saber hacer como docente donde se origina la identidad y es la formación que recibe un profesor universitario la que transforma esta construcción personal (Michavilla, 2017). Por tanto, en la construcción de una identidad docente han de analizarse las concepciones que el docente tiene sobre su propia disciplina, las que tiene sobre el aprendizaje y la dimensión emocional de su desarrollo profesional.

\section{DISEÑO METODOLÓGICO DE LA INVESTIGACIÓN}

La investigación, dentro del Proyecto antes mencionado, emplea un enfoque que combina distintas técnicas e instrumentos en distintas fases: un cuestionario de preguntas abiertas al inicio de la formación, el análisis de las producciones en forma de comunicación a las Jornadas de Docencia Universitaria, un cuestionario tipo Likert, el análisis de caso que incluye la observación de las clases de diez profesores seleccionados, entrevistas en profundidad, y la valoración que hacen los estudiantes de las innovaciones implementadas por los docentes que han participado en el programa FIDOP. No se trata con esto simplemente de una yuxtaposición de enfoques, sino de un intento de lograr la complementariedad de los datos cuantitativos y cualitativos, estableciendo un equilibrio entre la relevancia y el rigor en la captura e interpretación de los datos (Cohen, Manion \& Morrison, 2017; Creswell, 2017).

Los resultados que presentamos aquí se basan en el análisis de los datos del cuestionario abierto, que abarca las concepciones de enseñanza en las categorías curriculares de metodología, contenidos y evaluación, y la dimensión profesional objeto del presente trabajo. El propósito del cuestionario de preguntas abiertas fue recopilar información, antes de iniciar el programa formativo del año 2017, sobre las concepciones que tenían los profesores de las categorías y subcategorías que se iban a analizar. Las preguntas fueron elaboradas a partir de los ítems de tres cuestionarios validados y ampliamente utilizados en investigaciones anteriores, como la versión española del ATI (Approaches Teaching Inventory) (Monroy, González-Geraldo y Hernández-Pina, 2015), el cuestionario sobre "Concepciones del profesorado universitario acerca de la ciencia y su aprendizaje" (Martínez Galaz y González Weill, 2014) y el INPECIP (Inventario de Creencias Científicas y Pedagógicas del Profesorado, Porlán, 1989). Este cuestionario fue administrado en la primera sesión del curso por el formador de referencia con la finalidad de conocer las ideas de los docentes antes de empezar el proceso formativo. Para la validación del instrumento se atendió al juicio de diez expertos en Ciencias de la Educación, formación del profesorado y metodología de investigación de 
las Universidades de Sevilla y Granada. Tras su validación, se realizó una prueba piloto con diez docentes universitarios de diferentes especialidades, desempeño académico y experiencia docente. El análisis de los resultados implicó la reformulación de todas las preguntas con menos de cuatro puntos en pertinencia y claridad, tomándose en consideración las formulaciones alternativas de los evaluadores.

Los participantes en la investigación han sido 49 profesores de la Universidad de Sevilla inscritos en la edición del programa para el curso 2017, 32 mujeres y 17 hombres, que desarrollan su docencia en distintas facultades y escuelas, de los cuales 23 afirmaron haber recibido alguna formación pedagógica. De ellos, 11 desarrollan su docencia en el área de conocimiento de Arquitectura e Ingenierías, 5 en Arte y Humanidades, 19 en Ciencias Sociales y Jurídicas, 11 en Ciencias de la Salud y 3 en Ciencias. Con respecto a la experiencia docente universitaria de los participantes, 21 sujetos tenían entre 0 y 5 años de servicio como docentes, 4 sujetos tenían entre 6 y 10 años de docencia, 9 sujetos estaban entre 11 y 15 años, y 15 sujetos tenían más de 15 años de experiencia docente. A destacar que 4 sujetos con más de 20 años de experiencia afirmaron no haber recibido ninguna formación pedagógica. El análisis de estas variables no se ha tenido en cuenta en el presente estudio, centrándonos en las concepciones del profesorado en general. Si bien, consideramos que, en estudios posteriores sobre el cambio de las concepciones tras la realización del programa formativo, pueden ser variables relevantes a tener en cuenta.

En el análisis de los datos se ha seguido una metodología de análisis del contenido, en la línea que plantean Polit \& Beck (2006) y Krippendorff (2002). El objetivo era elaborar y categorizar unidades de significados a partir de inferencias válidas y replicables. Para gestionar tal tarea, nos hemos servido del programa Atlas.ti versión 8. Cada uno de los nueve investigadores implicados en el proyecto ha realizado una codificación por separado y posteriormente se han discutido los resultados para establecer consensos. Este enfoque de triangulación múltiple para la codificación de unidades de contenido pretende maximizar la validez del proceso (Patton, 2002).

En el presente trabajo vamos a realizar un análisis cuantitativo para obtener los porcentajes de unidades de información correspondientes a la dimensión profesional en la triple vertiente de concepciones sobre la propia disciplina, concepciones del aprendizaje y concepción de la identidad profesional docente. Además, realizaremos un análisis cualitativo de las respuestas al cuestionario dadas por los participantes a través del análisis del discurso (Van Dijk, 2016), y basándonos en una serie de unidades de información a modo de fragmentos discursivos paradigmáticos, que de alguna manera ejemplifican los distintos valores en la hipótesis de progresión 
del sistema de categorías. Estas unidades de información paradigmáticas o fragmentos de discurso representativos son identificadas mediante una codificación con los siguientes elementos (Tabla 1).

Tabla 1

Códigos para identificar las unidades de información

\begin{tabular}{|c|c|c|c|}
\hline \multirow{9}{*}{$\begin{array}{l}\text { SI1- Curso de } \\
\text { Iniciación } 1 \\
\text { SI2- Curso de } \\
\text { Iniciación } 2 \\
\text { SI3- Curso de } \\
\text { Iniciación } 3 \\
\text { SI4- Curso de } \\
\text { Iniciación } 4\end{array}$} & \multirow{9}{*}{$\begin{array}{l}\text { ART- Artes } \\
\text { ARQ- Arquitectura } \\
\text { ING- Ingeniería } \\
\text { MED- Medicina } \\
\text { ECO-Economía } \\
\text { POD-Podología } \\
\text { EDU- Educación } \\
\text { HIS- Historia } \\
\text { LEN- Lengua } \\
\text { MAT- Matemáticas }\end{array}$} & \multirow{9}{*}{$\begin{array}{l}\text { ENF- Enfermería } \\
\text { PER-Periodismo } \\
\text { FIS- Física } \\
\text { FIL- Filología } \\
\text { GEO- Geografía } \\
\text { FAR- Farmacia } \\
\text { FISIO- Fisioterapia }\end{array}$} & \multirow{4}{*}{$\begin{array}{l}\text { CU- Cuestionario } \\
\text { M- Mujer (Seguido } \\
\text { de edad) } \\
\text { H- Hombre } \\
\text { (Seguido de edad) }\end{array}$} \\
\hline & & & \\
\hline & & & \\
\hline & & & \\
\hline & & & FPN- Formación \\
\hline & & & Pedagógica No \\
\hline & & & (Seguido de años) \\
\hline & & & FPS- Formación \\
\hline & & & $\begin{array}{l}\text { Pedagógica Sí } \\
\text { (Seguido de años) }\end{array}$ \\
\hline
\end{tabular}

Para gestionar el tratamiento de las unidades de información se ha utilizado un sistema de categorías apriorístico (Tabla 2) que contempla una hipótesis de progresión con tres valores (Martínez y Martínez, 2012).

Tabla 2

Sistema de categorías e hipótesis de progresión

\begin{tabular}{|c|c|c|c|}
\hline $\begin{array}{l}\text { Categoría de } \\
\text { análisis }\end{array}$ & $\begin{array}{c}\text { Valor } 1 \\
\text { Modelo centrado } \\
\text { en la enseñanza }\end{array}$ & $\begin{array}{c}\text { Valor } 2 \\
\text { Modelo de } \\
\text { transición }\end{array}$ & $\begin{array}{c}\text { Valor } 3 \\
\text { Modelo centrado } \\
\text { en el aprendizaje }\end{array}$ \\
\hline $\begin{array}{l}\text { Concepciones } \\
\text { sobre la propia } \\
\text { disciplina -nivel } \\
\text { epistemológico } \\
\text { (CPD) }\end{array}$ & $\begin{array}{l}\text { Concepción } \\
\text { absolutista del } \\
\text { conocimiento, } \\
\text { que se concibe } \\
\text { como una verdad } \\
\text { incuestionable y } \\
\text { estática. }\end{array}$ & $\begin{array}{l}\text { Crisis de la } \\
\text { concepción } \\
\text { absolutista. } \\
\text { Adquiere } \\
\text { importancia la } \\
\text { historia de la } \\
\text { disciplina y sus } \\
\text { implicaciones } \\
\text { sociales. }\end{array}$ & $\begin{array}{l}\text { Concepción } \\
\text { relativista del } \\
\text { conocimiento } \\
\text { basada en la } \\
\text { evolución histórica } \\
\text { y en el valor social } \\
\text { de la disciplina. }\end{array}$ \\
\hline
\end{tabular}




\begin{tabular}{|c|c|c|c|}
\hline $\begin{array}{l}\text { Categoría de } \\
\text { análisis }\end{array}$ & $\begin{array}{c}\text { Valor } 1 \\
\text { Modelo centrado } \\
\text { en la enseñanza }\end{array}$ & $\begin{array}{c}\text { Valor } 2 \\
\text { Modelo de } \\
\text { transición }\end{array}$ & $\begin{array}{c}\text { Valor } 3 \\
\text { Modelo centrado } \\
\text { en el aprendizaje }\end{array}$ \\
\hline $\begin{array}{l}\text { Concepciones } \\
\text { sobre el } \\
\text { aprendizaje (CA) }\end{array}$ & $\begin{array}{l}\text { Los estudiantes no } \\
\text { tienen ideas sobre } \\
\text { los contenidos y } \\
\text { las que tienen son } \\
\text { erróneas. Aprender } \\
\text { es apropiarse del } \\
\text { conocimiento } \\
\text { científico o } \\
\text { sustituir las ideas } \\
\text { erróneas por el } \\
\text { conocimiento } \\
\text { verdadero. }\end{array}$ & $\begin{array}{l}\text { Los estudiantes } \\
\text { sí tienen ideas } \\
\text { y modelos } \\
\text { mentales, aunque } \\
\text { el aprendizaje se } \\
\text { produce por la } \\
\text { transmisión del } \\
\text { conocimiento } \\
\text { o intentos } \\
\text { espontaneístas por } \\
\text { descubrimiento. }\end{array}$ & $\begin{array}{l}\text { Aprender es hacer } \\
\text { evolucionar los } \\
\text { sistemas de ideas } \\
\text { de los estudiantes } \\
\text { hacia niveles de } \\
\text { conocimiento } \\
\text { de mayor } \\
\text { complejidad. }\end{array}$ \\
\hline $\begin{array}{l}\text { Identidad } \\
\text { profesional } \\
\text { docente (IPD) }\end{array}$ & $\begin{array}{l}\text { Se identifica } \\
\text { profesionalmente } \\
\text { con su disciplina de } \\
\text { referencia. }\end{array}$ & $\begin{array}{l}\text { La docencia } \\
\text { comienza a } \\
\text { formar parte } \\
\text { de la identidad } \\
\text { profesional. }\end{array}$ & $\begin{array}{l}\text { Se siente } \\
\text { identificado con } \\
\text { su desarrollo } \\
\text { profesional como } \\
\text { docente. }\end{array}$ \\
\hline
\end{tabular}

\section{ANÁLISIS DE LOS RESULTADOS}

\section{Las concepciones sobre la propia disciplina (CPD)}

En esta dimensión de análisis encontramos un 62.95\% unidades de información de valor 1 , un $18.55 \%$ de valor 2 y un $18.55 \%$ de valor 3. Por tanto, hay un claro predominio del valor inicial de la hipótesis de progresión que representa una concepción absolutista del conocimiento, en el que se otorga escaso interés a su evolución histórica y que tiende a una identificación con la verdad científica. Generalmente, se atribuye una gran importancia a la disciplina y se tiene poco en cuenta su trascendencia social. 


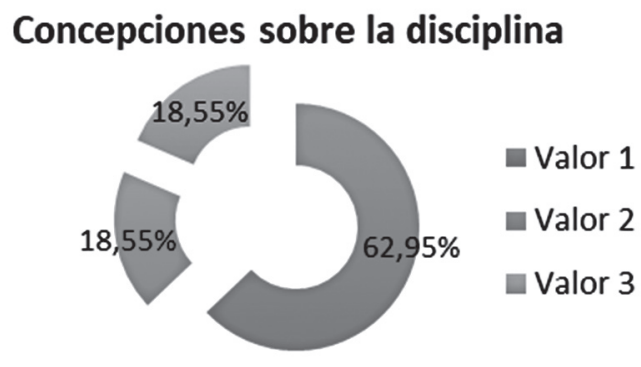

Figura 1. Concepciones sobre la propia disciplina

Algunos ejemplos de unidades de información correspondientes a este valor pueden ser:

Los contenidos de las asignaturas que imparto tratan precisamente de encontrar esta verdad a través de teorías, leyes, hipótesis y experimentación (SI4.48.FAR.CU.M.37.FPN.5).

En mi opinión mi área de conocimiento está muy basada en los resultados de la investigación científica básica, y está poco relacionada con la evolución histórica del conocimiento, a la que dedico habitualmente uno o dos temas (SI4.4.MED.CU.H.52.FPN.34).

Las matemáticas son imprescindibles en nuestra vida... no son creencias, sino resultados... En cada época de la historia, los descubrimientos científicos del momento se basan en avances de las matemáticas (SI3.46.MAT.CU.M.31.FPS.4).

En el valor 2 de la hipótesis de progresión, se admite la complejidad del conocimiento y la posibilidad de distintas perspectivas epistemológicas:

Mi disciplina, como parte de las humanidades, reconoce la complejidad del conocimiento actual, no habiendo una única solución en cada caso (SI2.38.ARQ.CU.H.29.FPN.1).

Trato de que mis alumnos relativicen, que tengan una visión crítica y conozcan las limitaciones de las supuestas verdades científicas... y las limitaciones de la modelización, de la medición de elementos subjetivos e incluso de los supuestamente objetivos (SI3.43.ECO.CU.M.50.FPS.23).

En este valor se contempla la historia y la evolución del conocimiento de la propia disciplina, pero se resignifica e interpreta de manera particular, pues la evolución no implica un carácter relativista del conocimiento, 
como construcción social e histórica, sino que se concibe con una visión teleológica, tendente y dirigida a un fin, la verdad, los avances científicos y el progreso:

Evolución histórica impresionante de las tecnologías de la construcción desde los romanos... los inventos desarrollados por los ingenieros en todos los campos son la base de la sociedad en la que vivimos... esto se lo repito continuamente mis alumnos (SI4.17.ARQ. CU.M.46.FPN.12).

En cuanto al valor social del conocimiento, se describe en términos de utilidad, eficacia y aportación al progreso. No se distingue para quién es útil y no hay perspectiva crítica, pues la sociedad se concibe como un todo armónico al que el conocimiento de la disciplina ofrece resultados. Así lo observamos en algunas unidades de información prototípicas que ofrecemos a continuación:

Creo que no somos conscientes de la cantidad de ventajas que ha supuesto la investigación fundamental en la mejora de las sociedades actuales (SI2.14.FIS.CU.M.33.FPN.3).

Creo que en el campo de conocimiento en el que trabajo se están haciendo avances que tienen repercusión en la sociedad y que resultan útiles (SI1.2.ING.CU.M.35.FPN.4).

A veces el valor social del conocimiento de la disciplina de referencia se entiende en el sentido mercantilista de la eficacia y la "satisfacción del cliente":

En cuanto al valor social de mi disciplina, es considerable porque importa la aceptación del usuario final del edificio, premia obtener métodos de construcción funcional, que afecte mínimamente a nivel de ruido, uso de materiales y gestión de los residuos (SI3.16.ARQ.CU.H.27. FPN.0).

El valor y la utilidad social innegable en la arquitectura que debe tener siempre en cuenta al usuario del edificio (SI4.49:ARQ.CU.M.48. FPN.21).

En el valor 3, encontramos una visión relativista del conocimiento, que se concibe como una construcción social e histórica. Veamos algunos ejemplos paradigmáticos: 
No hay verdad científica inamovible, el hecho histórico es así hasta que algún hallazgo o documento plantee una revisión... en la Historia de la comunicación hay muchas verdades que se han ido modificando con el paso del tiempo al revisarse la perspectiva desde la que se habian enunciado (SI1.36.PER.CU.M.42.FPN.11).

Cuestionamos constantemente las verdades científicas, ese cuestionamiento constituye una forma de construir la verdad científica (SI4.41.GEO.CU.H.35.FPS.8).

No creo en una verdad científica única y absoluta. Entiendo la economía como una ciencia social cuyos conceptos y teorías están influenciados por valores y cosmovisiones de quienes las definen, existiendo muy distintos enfoques y escuelas, algunas dominantes y otras minoritarias o heterodoxas (SI3.34.ECO.CU.M.47.FPN.20).

Con respecto al valor social del conocimiento, en este valor 3 aparecen elementos relacionados con la responsabilidad y el compromiso social de la profesión. Así, encontramos respuestas del tipo:

La profesión de periodista debe recuperar el compromiso social, su responsabilidad para con la ciudadanía y permitir la formación de una opinión pública crítica... (SI3.13.PER.CU.M.39.FPS.15).

Esta disciplina, al igual que la Historia, la Sociología, el Medio Ambiente o la Economía, debe ser una herramienta de transformación social hacia una sociedad más justa e igualitaria (SI4.41.GEO.CU.H.35. FPS.8).

\section{Concepciones sobre el aprendizaje de los estudiantes (CA)}

En esta categoría un $80.95 \%$ de las unidades de información se sitúan en el valor 1 de la hipótesis de progresión en relación con las concepciones sobre el aprendizaje (CA), un $19.04 \%$ está en el valor 2 y ningún docente se sitúa en el valor 3 , es decir, en un modelo de enseñanza centrado en el aprendizaje de los alumnos en el que el docente guía el progreso de las ideas de los estudiantes hacia formulaciones más complejas. 


\section{Concepciones sobre el aprendizaje}

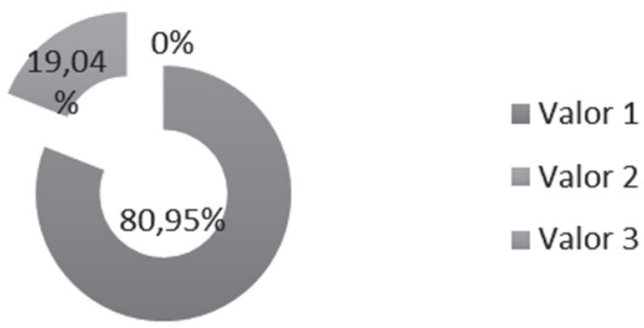

Figura 2. Concepciones sobre el aprendizaje

En el valor 1, los docentes centrados en la enseñanza consideran que los alumnos por sí solos no aprenden porque no tienen recursos, su nivel de partida es cero y el conocimiento que adquieren por sí mismos es erróneo y debe ser corregido. Aunque durante la clase se permita la participación del alumnado, con algún tipo de debate o trabajo cooperativo, el docente piensa que esto no conduce a un aprendizaje correcto, por lo que es necesario complementar con la explicación teórica. Valgan como ejemplos las siguientes unidades de información:

Pienso que el que los alumnos por sí solos se enfrenten con sus conocimientos en primer lugar a intentar poner en pie y razonar en grupo un caso (siempre con mi guía para orientar si van bien encaminados o no), y que posteriormente yo corrija errores y refuerce lo acertado para toda la clase (SI4.9.MED.CU.M.31.FPN.3).

Además, con el pequeño debate hablan unos con otros y argumentan, lo que creo que les ayuda a ver otros puntos de vista. Sin embargo, normalmente no están en lo correcto, lo que me lleva a tener que realizar alguna explicación teórica para poder llevar a cabo esta práctica (SI2.14.FIS.CU.M.33.FPN.3).

En estos casos prevalece un modelo centrado en la enseñanza porque en ninguna de las situaciones los alumnos tienen el protagonismo a la hora de construir el conocimiento. La participación del alumnado es una cuestión puramente formal, una concesión a lo pedagógicamente correcto, pero no se considera algo sustancial en el proceso de enseñanza-aprendizaje.

A menudo la opinión que tiene el docente sobre sus alumnos condiciona sus creencias sobre el aprendizaje, pues piensan, mayoritariamente, que el alumnado es apático per se, no está motivado, no tiene curiosidad, solo 
les interesa la nota del examen y no son capaces de relacionar conceptos sin la intervención del profesor. Además, al profesor o profesora centrado en la enseñanza le preocupa trasmitir bien, explicar bien, que el alumno desarrolle y ejecute adecuadamente lo enseñado; ser respetado, tener autoridad, marcar las distancias, que haya silencio en las clases.

Por parte de los alumnos, se observa una apatía inicial, sobre todo en el arranque de un tema nuevo. Es una reacción lógica, y creo que debida a la inseguridad ante unos conceptos nuevos, si todavía no están asentados los anteriores. Además, existe una dificultad natural de comprensión, que es más destacable en alumnos de primer curso (SI1. MAT.CU.H.51.FPS.19).

Me frustra mucho cuando tras intentar explicar algo de más de 5 formas diferentes para que lo entiendan bien, la única pregunta que me hacen es si entraría eso en el examen (SI4.ENF.CU.M.4.FPS.1).

Solo les interesa la nota, no se sienten parte activa del aprendizaje, están educados para recibir y consumir, no valoran la importancia del saber, creen que acumular datos es en lo que consiste la profesión (SI4.40.MED.CU.M.58.FPN.19).

Cuando la clase está revoltosa, cuando a veces estoy un poco de mal humor (que no cansado) o por romper la dinámica, a veces suelto un manotazo en un pupitre que genera un ruido seco e impactante. Me sirve para enviar un mensaje de autoridad, que suele contrastar con mi disposición afable. Me sirve para marcar las distancias ante mi apariencia juvenil (para que no se confíen). Cuando empieza la clase, me gusta que haya silencio. Después no me importa que hablen, discutan... (SI4.GEO.CU.H.35.FPS.8).

A veces se intenta suplir la monotonía de la explicación tradicional con algún elemento tecnológico, pero finalmente el resultado es el mismo.

Lo que menos me gusta de las clases es mi dependencia respecto a las diapositivas. Estas además, son muy "largas", llenas de texto. En realidad, el powerpoint lo hago para mí y no tanto para ellos; lo hago para no tener que llevar notas o memorizarlo todo. Me es más cómodo (SI1.27.ECO.CU.M.55.FPN.23).

Encontramos algunas unidades de información que denotan que los profesores y profesoras, a pesar de lo dicho hasta ahora, son capaces de tomar conciencia de las limitaciones de su práctica, abriendo la posibilidad de iniciar un cambio profesional. 
Las asignaturas que imparto no suelen ser atractivas para los alumnos y su contenido es árido y poco practicable en su profesión asistencial. (...) Es un contenido de historia y legislación que no es nada atractivo para un futuro profesional clínico. En ocasiones reconozco que hasta a mi me resultan pesados algunos aspectos, asi que los comprendo más a ellos (SI3.10.POD.CU.H.39.FPN.3).

Si cuando les pregunto no contestan nada, me siento impotente por no saber si no me están entendiendo, o se están aburriendo porque les parece muy fácil (SI3.MAT.CU.M.31.FPS.4).

En el valor 2 de nuestra hipótesis de progresión encontramos unidades de información que ofrecen transiciones en las que los profesores y profesoras dan mayor participación al alumnado y favorecen el paso desde una clase magistral a otra más activa. En tal caso consideran que los alumnos tienen ciertas ideas y se valora que a través de ellas lleguen a determinadas conclusiones, con actividades que permiten una reflexión crítica, aunque siempre prevalece la trasmisión teórica de los contenidos para garantizar el proceso de enseñanza-aprendizaje. En este sentido, podemos observar el ejemplo de un profesor de Geografía:

Sí, lo que hago es preguntar oralmente algunas cuestiones sobre los contenidos a dar para saber si ya han adquirido los mismos en otras asignaturas afines y hasta qué nivel de profundización y tengo en cuenta la información obtenida para intentar partir de ahí (SI1.26.GEO. CU.M.39.FPS.14).

La siguiente unidad de información muestra el interés por explorar las ideas de los alumnos, aunque al cabo solo se pretende comprobar si estos "saben o no saben", concibiendo el aprendizaje no como un proceso evolutivo sino como una adquisición que no admite gradualidad.

Empiezo la clase tipo "clase magistral". Pregunto con muchísima frecuencia a los alumnos, antes, durante y después de clase. Cuando no hayvoluntari@s,preguntodirectamentealosalumn@s.Cuandopregunto antes de explicar, la idea fundamental es saber sus conocimientos sobre el tema; sus opiniones. Esto me orienta mucho a la hora de plantear las cuestiones. Durante la clase pregunto también bastante; el objetivo es saber si se está comprendiendo la explicación; qué opinión tienen los alumnos respecto a lo que se explica; si lo comparten o no. También me ayuda a hacer la clase más amena, menos cansada, más distendida (SI1.27.ECO.CU.M.55.FPN.23). 
En determinadas dinámicas de aula, en las que se valoran las ideas de los estudiantes sobre los contenidos, el papel del docente queda elidido pues no se concibe que pueda ayudar a sus alumnos a evolucionar hacia niveles de formulación de los contenidos más complejos, más bien se pretende que estos aprendan por descubrimiento. Un profesor de Enfermería dice:

Porque pienso que lo que descubre uno por sí mismo es un conocimiento más sólido que si les llega por transmisión directa del profesor. Si el alumno interioriza el hecho de razonar y relacionar ideas, hechos y situaciones; tendrá una herramienta muy eficaz para el aprendizaje futuro (SI2.12.ENF.CU.M.54.FPS.11).

Además, encontramos que una mayor valoración de la participación de los alumnos hace sentirse el docente considerado y reconocido. Ahora bien, no conciben la participación como una forma de aprender, sino más bien como una concesión, un dispositivo pedagógico, que finalmente necesita la intervención del docente "dando" el temario.

Las preguntas: "obliga" a los alumnos a pensar, a tomar conciencia de su nivel de conocimiento, a posicionarse teórica e ideológicamente respecto a los problemas que se plantean, a contrastar sus ideas/ conocimientos y opiniones con las de sus compañer@s, a tomar una postura más activa ante el conocimiento y la clase. En resumen, les ayuda/fuerza a madurar, y creo que esto lo agradecen porque se sienten considerados, sienten que son más escuchados y respetados y que son tratados como lo que son: personas adultas. Obviamente, hay que buscar un equilibrio: no podemos estar todo el tiempo debatiendo porque hay que "dar" contenidos; hay que avanzar en el temario y no siempre se tiene tiempo para debatir todas las "cosas" que son susceptibles de ser debatidas (SI1.27.ECO.CU.M.55.FPN.23).

\section{La identidad profesional docente}

En cuanto a las concepciones sobre la identidad profesional, encontramos $51.16 \%$ de valor 1, 26.7\% de valor 2 y $22.09 \%$ de valor 3 en la hipótesis de progresión. 


\section{Identidad profesional docente}

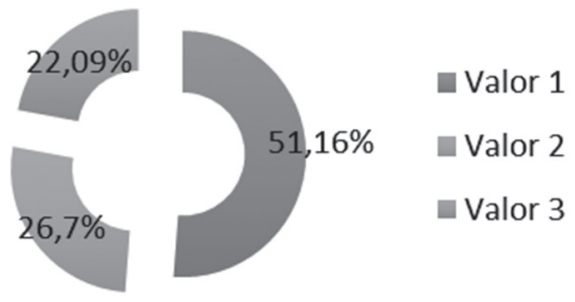

Figura 3. Identidad profesional docente

En el valor 1 predomina una identificación con la investigación, como vocación y campo profesional preferente:

La investigación es el campo en el que me encuentro más cómoda (SI4.30.PER.CU.M.39.FPS.10).

Cuando les pedimos que cuantifiquen qué tiempo dedican a las distintas tareas universitarias, encontramos:

Docencia: 20\%, Investigación: 80\% (SI3.45.ECO.CU.M.34.FPN.5).

La docencia se percibe como una obligación, y de ahí deriva cierta preocupación por hacerlo lo mejor posible, pues se le reconoce un valor social:

He dedicado más tiempo a la investigación, aunque cuando he tenido docencia he intentado prepararme lo mejor posible las clases y que los alumnos aprendieran (SI2.46.MAT.CU.M.31.FPS.4).

Me identifico en mayor medida con la investigación porque es lo que verdaderamente me apasiona de la profesión universitaria. No obstante, considero que lo que mayor incidencia social tiene en la actividad de un PDI es la docencia (SI1.28.HIS.CU.H.34.FPS.8).

Cuando se valora la docencia, esta se concibe como una tarea rutinaria, en la que el alumnado cumple un papel subalterno, como destinatario de los resultados de la labor investigadora del profesor:

La investigación es mi verdadera vocación... la docencia me permite trasladar a los alumnos lo último en relación con los avances en la disciplina (SI3.20.GEO.CU.H.48.FPS.20). 
Yo me dediqué a la Universidad porque me gustaba investigar: si hubiera querido ser docente me hubiera tirado por secundaria. La docencia la he ido descubriendo gracias al trabajo rutinario (las clases, los alumnos) (SI1.25.FIL.CU.M.40.FPN.9).

Incluso, en algún caso, la docencia se concibe como una oportunidad para seguir experimentando con la propia disciplina de referencia:

Si un artista quiere que su trabajo sea potente, no basta con pintar bien, tiene que saber transmitir el mensaje... en este sentido me siento completa al realizar tareas docentes, porque percibo que estoy aprendiendo muchas cosas que quizá no hubieran surgido estando en el estudio trabajando (SI2.1.ART.CU.M.26.FPN.O).

En el valor 2 de la hipótesis de progresión empezamos a encontrar unidades de información que denotan una valoración de la docencia como actividad profesional interesante y motivadora, aunque lastrada por distintos condicionantes como la escasa valoración académica, la falta de tiempo, el desgaste psicológico del trato con el alumnado, etc.

Debo fraguarme un currículum, me identifico en mayor medida con la labor investigadora. No obstante siempre he estado muy interesada en el ejercicio docente, sea cual sea el nivel (secundaria/universidad)..., me interesa no solo dar clase, sino también la investigación relacionada con la innovación docente (SI3.32.LEN.CU.M.25.FPS.2).

La docencia me gusta mucho por el contacto con mis alumnos, porque me permite seguir curioseando sobre las materias que imparto... pero me genera mucho desgaste, tanto físico como emocional... últimamente estoy intentando distanciarme un poco de todas estas cuestiones a nivel sentimental... por otra parte a mí lo que me gusta es investigar, buscar, indagar, encontrar respuestas y escribir (SI1.25.FIL. CU.M.40.FPN.9).

A la docencia es a lo que dedico más tiempo con diferencia, la investigación queda en un segundo plano por falta de tiempo (SI.1.26. GEO.CU.M.39.FPS.14).

A veces se pone de manifiesto cómo "lo que se hace" genera identidades, o cómo la praxis, aunque originariamente se experimentase como una obligación impuesta por las circunstancias académicas, acaba contribuyendo a la construcción de un discurso sobre la identidad profesional docente: 
Intento equilibrar entre docencia e investigación porque me gustan ambas actividades y porque una y otra se nutren mutuamente. La docencia es una obligación que vivo como compromiso, es el sentido de la Universidad y la principal dimensión de utilidad social de nuestro trabajo. La investigación me acerca a comprender el mundo y participar en el debate sobre qué está pasando, nutre mi docencia a la vez que las clases nutren mi investigación a partir de dudas y debates que aparecen en las clases (SI3.34.ECO.CU.M.47.FPN.20).

Finalmente, en el valor 3 de la hipótesis de progresión, encontramos una clara identificación con la docencia, como actividad motivadora y útil desde el punto de vista social y académico:

Me identifico más con la docencia porque sin lugar a dudas es lo que más me gusta. De hecho inicialmente no tenía interés en hacer el doctorado, sino en prepararme secundaria (SI2.11.ENF.CU.H.38.FPS.5).

Tengo al público sujeto de mi esfuerzo diario delante, noto cómo cambia (todavía de forma limitada) su manera de ver el mundo, hablo con ellos y ellas, me siento que estoy ganándome el sueldo. Cosa que no es tan fácil de percibir con los lectores potenciales de papers y comunicaciones (SI4.41.GEO.CU.H.35.FPS.8).

\section{DISCUSIÓN Y CONCLUSIONES}

En líneas generales, se confirman las evidencias proporcionadas por estudios anteriores en diversos aspectos, pues, en la muestra analizada se pone de manifiesto la prevalencia del modelo de enseñanza tradicional y la falta de conocimiento pedagógico experto (Amundsen \& Wilson, 2012). Sin embargo, nuestra investigación aporta una caracterización de las concepciones del profesorado universitario en unas dimensiones que se han considerado clave para la conformación de un modelo didáctico centrado en el aprendizaje de los alumnos.

Con respecto a la concepción del aprendizaje (CA), los participantes en esta investigación, en coherencia con un enfoque centrado en su propia enseñanza, la conciben como un proceso de transmisión del conocimiento del profesor a sus alumnos. A veces observamos ciertas concesiones formales a una práctica más renovada, que toma la forma de la incorporación de nuevas tecnologías o de dispositivos que permiten la participación de los alumnos, aunque esta se considera como un elemento puramente formal, no como una forma de aprender. Tanto en un caso como en otro, el papel 
del alumnado es de mero receptor, pues su conocimiento necesita de la corrección del profesor. En algunas unidades de información los docentes muestran una idea estereotipada de los alumnos (apatía, falta de interés, obsesión por el examen) que obstaculiza la posibilidad de otorgarles carta de naturaleza en el proceso de enseñanza-aprendizaje. Incluso, en algún caso, esto provoca una actitud defensiva por parte del docente, que coloca de esta forma los obstáculos fuera del ámbito de su actuación, haciendo muy difícil el proceso de toma de conciencia de las propias limitaciones y, por tanto, las opciones de cambio y desarrollo profesional.

No obstante, aparecen unidades de información que denotan un interés por parte del docente por trabajar con las ideas del alumnado. Sin embargo, la exploración de estas, al comenzar una secuencia, se concibe como el intento de averiguar si "sabe o no sabe. Y, aunque algunos se refieren a la necesaria gradualidad del proceso, en el fondo el conocimiento no se entiende como una reformulación progresiva de las ideas.

En relación con la concepción de la propia disciplina (CPD), predomina una concepción absolutista del conocimiento y una visión positivista de la verdad científica. La evolución histórica de la disciplina no tiene carácter relativista, sino más bien dirigida a un fin, el progreso material. El valor social adquiere un tono un tanto ingenuo, utilitarista y acrítico, pues se cree que el conocimiento está al servicio de una sociedad entendida como un todo armónico y sin conflictos. Estos resultados son coherentes con las evidencias proporcionadas por estudios anteriores que señalan cómo en el ámbito universitario se prioriza la especialización en la disciplina y la investigación en detrimento de la docencia (Postareff, 2007; Zabalza et al., 2014). Las formulaciones de transición nos llevan a una visión más evolutiva y relativista del conocimiento y un valor social ligado al compromiso y la responsabilidad que facilitarían la adquisición de competencias profesionales en el alumnado.

Para la categoría relativa a la identidad profesional docente (IPD), entendida como dimensión resultante de las categorías anteriores (Monereo, 2014), se constata que el profesorado centrado en la enseñanza no la desarrolla porque solo se identifica con su disciplina de referencia y su labor investigadora, y refiere la existencia de obstáculos externos que impiden la definición de una identidad profesional docente. Algunos profesores afirman que, aunque se identifican con la investigación y siempre han considerado la docencia como una mera obligación académica, la praxis ha terminado generando identidad profesional docente (Bourdieu, 2002), poniendo de manifiesto que es posible su construcción o reconceptualización. 
En síntesis, la caracterización de las concepciones de los profesores universitarios que proporciona este estudio, aporta un sistema de categorías emergentes o empírico, en la línea de la grounded theory (Lambert, 2019) que esperamos pueda ser útil tanto para el análisis de las evoluciones de los participantes en el programa formativo FIDOP, como para el diseño de otros programas de formación del profesorado universitario, permitiendo un desarrollo profesional a partir de las transiciones halladas y hacia un modelo de enseñanza más centrado en los estudiantes (Paricio Royo et al., 2019).

Nuestro estudio señala la necesidad de atender, en los programas formativos del profesorado universitario, a la desconstrucción de creencias no reflexionadas, acríticas, que suponen un obstáculo para el progreso de los participantes. Particularmente, el aprendizaje reflexivo y la metacognición podrían contribuir a la toma de conciencia sobre cómo aprendemos y, por tanto, a comprender mejor cómo aprenden nuestros alumnos. En cuanto a la concepción de la propia disciplina convendría incluir, en los programas formativos, actividades de reflexión sobre la construcción social e histórica del conocimiento como forma de cuestionar la concepción absolutista del mismo. Por último, consideramos que la práctica docente reflexiva e investigativa, contribuye a la construcción de la identidad profesional docente y, por tanto, habría que poner mayor énfasis en las actividades de investigación sobre la propia práctica docente. 


\section{REFERENCIAS BIBLIOGRÁFICAS}

Amundsen, Ch. \& Wilson, M. (2012). Are We Asking the Right Questions? A Conceptual Review of the Educational Development Literature in Higher Education. Review of Educational Research, 82(1), 90-126. https://doi. org/10.3102/0034654312438409

Bain, K. (2004). Lo que hacen los mejores profesores universitarios. Universidad de Valencia.

Bourdieu, P. (2002). Las reglas del arte. Génesis y estructura del campo literario. Anagrama.

Cohen, L., Manion, L., \& Morrison, K. (2017). Research methods in Education. Routledge.

Conde-Jiménez, J. y Martín-Gutiérrez, A. (2016). Potencialidades y necesidades de mejora en la formación de profesores noveles universitarios. Revista Electrónica de Investigación Educativa, 18(1), 140-152.

Creswell, J.W. (2017). Qualitative inquiry \& research design: Choosing among five approaches. Sage.

De Alba N. y Porlán, R. (2017). La metodología de enseñanza. En R. Porlán (Coord.), Enseñanza Universitaria. Cómo mejorarla (pp. 3753). Morata.

Drago, C., Espejo, R. y González, J. (2015). ¿Qué significa ser profesor universitario? Un enfoque a través de declaraciones de filosofía de enseñanza-aprendizaje. Docencia Universitaria, 16, 87-102.

Gibbs, G., \& Coffey, M. (2004). The impact of training of university teachers on their teaching skills, their approach to teaching and the approach to learning of their students. Active learning in Higher
Education, 5(1), 87-100. https://doi. org/10.1177/1469787404040463

Krippendorff, K. (2002). Metodología de análisis de contenido. Teoría y práctica. Paidós.

Lambert, M. (2019). Grounded Theory. In Mike Lambert (Ed.). Practical Research Methods in Education: An Early Researcher's Critical Guide, Routledge.

Martín del Pozo, R. M., Pineda-Alfonso, J.A. y Duarte, O. (2017). La formación docente del profesorado universitario. En R. Porlán (Coord.). Enseñanza universitaria. Cómo mejorarla (pp. 2336). Morata.

Martínez, C. y Martínez, V.G. (2012). El conocimiento escolar y las Hipótesis de Progresión: algunos fundamentos y desarrollos. Nodos y nudos, 32, 50-64. https://doi. org/10.17227/01224328.1799

Martínez Galaz, C.P., y González Weil, C.U. (2014). Concepciones del profesorado universitario acerca de la ciencia y su aprendizaje y cómo abordan la promoción de competencias científicas en la formación de futuros profesores de Biología. Enseñanza de las Ciencias, 32(1), 51-81. https://doi. org/10.5565/rev/ensciencias. 852

Medina Moya, J.L., y Jarauta Borrasca, B. (Coords) (2013). Enseñanza y aprendizaje en la Educación Superior. Síntesis.

Michavilla, F. (2017). El perfil del buen docente. Cuadernos de Pedagogía 476, 60-66.

Monereo, C. (2014). Enseñando a enseñar en la Universidad. La formación del profesorado basada en incidentes críticos. Octaedro. 
Monroy, F., González-Geraldo, J., \& Hernández-Pina, F. (2014). A psychometric analysis of the Approaches to Teaching Inventory (ATI) and a proposal for a Spanish version (S-ATI-20). Anales De Psicología / Annals of Psychology, 31(1), 172-183. https://doi.org/10.6018/ analesps.31.1.190261

Schön, D. (1992). La formación de profesionales reflexivos. Hacia un nuevo diseño de la enseñanza y el aprendizaje en las profesiones. Paidós.

Stenhouse, L. (1985). Investigación y desarrollo del curriculum. Morata.

Paricio Royo, J.; Fernández March, A., y Fernández Fernández, I. (coords.) (2019). Marco de desarrollo académico docente. Un mapa de la buena docencia universitaria basado en la investigación. REDU, Red de Docencia Universitaria.

Patton, M. Q. (2002). Qualitative Research \& Evaluation Methods. [3. ed.]. Sage Publications.

Polit, D.F., \& Beck, C. (2006). Essentials of nursing research methods, appraisal, and utilization. Lippincott Williams \& Wilkins.

Porlán, R., y Rivero, A. (1998). El conocimiento de los profesores. Díada.

Porlán, R. (1989). Teoría del conocimiento, teoría de la enseñanza y desarrollo profesional. Las concepciones epistemológicas de los profesores. [Tesis Doctoral]. Universidad de Sevilla.

Postareff, L; Lindblom, S., \& Nevgi, A. (2007). The effect of pedagogical training on teaching in Higher Education. Teaching and Teacher Education, 23, 557-571. https://doi. org/10.1016/j.tate.2006.11.013

Van Dijk, T. (2016). Estudios Críticos del Discurso: Un enfoque sociocognitivo. Discurso \& Sociedad, 10(1), 137-162.

Vázquez, J., Solís, E. y Porlán, R. (2017). Introducción. En R. Porlán (Coord.). Enseñanza universitaria. Cómo mejorarla, (pp. 17-20). Morata.

Zabalza, M.A.; Cid, A., y Trillo, F. (2014). Formación docente del profesorado universitario. El difícil tránsito a los enfoques institucionales. Revista Española de Pedagogía, 257, 39-54. 


\section{PERFIL ACADÉMICO Y PROFESIONAL DE LOS AUTORES}

José A. Pineda-Alfonso. ORCID: http://orcid.org/0000-0002-6379-5686

Profesor Asociado de Didáctica de las Ciencias Sociales en la Facultad de Ciencias de la Educación de la Universidad de Sevilla (España). Líneas de investigación: enseñanza de las Ciencias Sociales, la formación del profesorado, la educación para la ciudadanía y la participación y la convivencia escolar. Email: apineda@us.es

Olga María Duarte Piña. ORCID: http://orcid.org/0000-0001-7341-6689

Profesora e investigadora en el departamento de Didáctica de las Ciencias Experimentales y Sociales de la Universidad de Sevilla. Miembro del Grupo DIE (Didáctica e Investigación Escolar). Líneas de investigaciones: innovación educativa, didáctica del patrimonio cultural y formación del profesorado. Email: oduarte@us.es

Fecha Recepción del Artículo: 26. Agosto. 2019

Fecha Modificación del Artículo: 30. Diciembre. 2019

Fecha Aceptación del Artículo: 10. Enero. 2020

Fecha Revisión para Publicación: 21. Enero. 2020 